\title{
An automatic strategy for adaptive tetrahedral mesh generation
}

\author{
R. Montenegro ${ }^{\text {a,* }}$, J.M. Cascón ${ }^{\text {b }}$, J.M. Escobar ${ }^{\text {a }}$, E. Rodríguez ${ }^{\text {a }}$, \\ G. Montero ${ }^{\text {a }}$ \\ ${ }^{a}$ University of Las Palmas de Gran Canaria, University Institute for Intelligent Systems \\ and Numerical Applications in Engineering, Spain. \\ ${ }^{\mathrm{b}}$ University of Salamanca, Department of Mathematics, Faculty of Sciences, Spain.
}

\begin{abstract}
This paper introduces a new automatic strategy for adaptive tetrahedral mesh generation. A local refinement/derefinement algorithm for nested triangulations and a simultaneous untangling and smoothing procedure are the main involved techniques. The mesh generator is applied to 3-D complex domains whose boundaries are projectable on external faces of a meccano approximation composed of cuboids. The domain surfaces must be given by a mapping between meccano surfaces and object boundary. This mapping can be defined by analytical or discrete functions. At present, we have fixed mappings with orthogonal, cylindrical and radial projections, but any other one-to-one projection may be considered.

The mesh generator starts from a coarse and valid hexahedral mesh that is obtained by an admissible subdivision of the meccano cuboids. The automatic subdivision of each hexahedron into six tetrahedra produces an initial tetrahedral mesh of the meccano approximation.

The main idea is to construct a sequence of nested meshes by refining only those tetrahedra with a face on the meccano boundary. The virtual projection of meccano external faces defines a valid triangulation on the domain boundary. Then a 3-D local refinement/derefinement is carried out so that the approximation of domain surfaces verifies a given precision. Once this objective is reached, those nodes placed on the meccano boundary are really projected on their corresponding true boundary, and inner nodes are relocated using a suitable mapping. As the mesh topology is kept during node movement, poor quality or even inverted elements could appear in the resulting mesh; therefore, we finally apply a mesh optimization procedure. The efficiency of the proposed technique is shown with several applications to complex objects.
\end{abstract}

Key words: Tetrahedral mesh generation, adaptive refinement/derefinement, nested meshes, mesh smoothing, mesh untangling, 3-D finite element method.

1991 MSC: 65M50, 65N50 


\section{Introduction}

In finite element simulation in engineering problems, it is crucial to automatically adapt the three-dimensional discretization to geometry and to solution. In the past, many authors have devoted great effort to solving this problem in different ways $[2,11,12,31]$, but automatic 3-D mesh generation is still an open problem. Generally, as the complexity of the problem increases (domain geometry and model), the methods for approximating the solution become more complicated. At present, it is well known that most mesh generators are based on Delaunay triangulation and advancing front technique. On the other hand, local adaptive refinement strategies are employed to adapt the mesh to singularities of numerical solution. These adaptive methods usually involve remeshing or nested refinement [14,17,18,27]. Another interesting idea is to adapt simultaneously the model and the discretization in different regions of the domain. A perspective on adaptive modeling and meshing is studied in [3]. The main objective of all these adaptive techniques is to achieve a good approximation of the real solution with minimal user intervention and low computational cost. For this purpose, the mesh element quality is also an essential aspect for the efficiency and numerical behaviour of finite element method. The element quality measure should be understood depending on the isotropic or anisotropic character of the numerical solution.

In this paper we present new ideas and applications of an innovative tetrahedral mesh generator which was introduced in [21,4]. This automatic mesh generation strategy uses no Delaunay triangulation, nor advancing front technique, and it simplifies the geometrical discretization problem for 3-D complex domains, whose surfaces can be mapped from a meccano face to object boundary. The main idea of the new mesh generator is to combine a local refinement/derefinement algorithm for 3-D nested triangulations [17] and a simultaneous untangling and smoothing procedure [7]. The resulting adaptive meshes have an appropriate quality for finite element applications.

At present, this idea has been implemented in ALBERTA code [30,29]. This software can be used for solving several types of 1-D, 2-D or 3-D problems with adaptive finite elements. The local refinement and derefinement can be done by evaluating an error indicator for each element of the mesh and it is based on element bisection. To be more specific, the newest vertex bisection method is implemented for 2-D triangulations [20]. Actually, ALBERTA has implemented an efficient data structure and adaption for 3-D domains which can be decomposed into hexahedral elements as regular as possible. Each hexahedron is subdivided into six tetrahedra by constructing a main diagonal and its projections on its faces, see Figure 1(a). The local bisection of the resulting tetrahedra is recursively carried out by using gen-

\footnotetext{
* Corresponding author.

Email address: rafa@dma.ulpgc.es (R. Montenegro).

URL: www.dca.iusiani.ulpgc .es/proyecto0507 (R. Montenegro).
} 
eral ideas of the longest edge [28] and the newest vertex bisection methods. The refinement of a given triangulation is performed by a recursive algorithm. In order to guarantee that this algorithm terminates in a finite number of iterations, several conditions on the initial mesh are necessary. Basically, the algorithm requires that the refinement edge of an element in the initial mesh is the same for all elements that share this edge. Details about the local refinement technique implemented in ALBERTA for two and three dimensions and restrictions on initial mesh are analyzed in $[30,17,20]$. This strategy works very efficiently for initial meshes obtained by subdivision of regular quadrilateral or hexahedral elements. In these cases, the degeneration of the resulting 2-D or 3-D triangulations after successive refinements is avoided. The restriction on the initial element shapes and mesh connectivities makes it necessary to develop a particular mesh generator for ALBERTA. In this paper, we summarize the main ideas introduced for this purpose. Obviously, our mesh generation technique could be developed in other codes. Moreover, these ideas could be combined with other types of local refinement/derefinement algorithms for tetrahedral meshes [14,18,27].

The meccano technique presents several advantages with respect to more traditional approaches, such as Delaunay triangulation or advancing front technique $[2,11,12,31]$. Delaunay triangulation requires a control in order to avoid slivers. Furthermore, the mesh conformity with the object boundary is not a trivial problem for complex geometry. On the other hand, advancing front technique requires a suitable surface triangulation. In addition, an appropriate definition of element sizes is demanded for obtaining good quality tetrahedra. Other technical difficulties appear when these two methods are applied to objects comprising different materials.

Our approach is based on the combination of several former procedures (refinement, derefinement, projection, untangling and smoothing) which are not in themselves new, but the overall integration is an original contribution. Authors have used them in different ways. Triangulations for convex domains can be constructed from a coarse mesh by using refinement/projection [29]. Adaptive nested meshes have been constructed with refinement/derefinement algorithms for evolution problems [8]. Large domain deformations can lead to severe mesh distortions, especially in 3-D. Mesh optimization is thus key for keeping mesh shape regularity and for avoiding a costly remeshing $[15,16]$. In traditional mesh optimization, mesh moving is guided by the minimization of certain overall functions, but it is usually done in a local fashion. In general, this procedure involves two steps [10,9]: the first is for mesh untangling and the second one for mesh smoothing. Each step leads to a different objective function. In this paper, we use the improvement proposed by [7], where a simultaneous untangling and smoothing guided by the same objective function is introduced.

Some advantages of our technique are that: surface triangulation is automatically constructed, the final 3-D triangulation is conforming with the object boundary, inner surfaces are automatically preserved (for example, interface between several 
materials), node distribution is adapted in accordance with the object geometry, and parallel computations can easily be developed for meshing the meccano pieces. Nevertheless, our procedure demands an automatic construction of the meccano approximation. In addition, an admissible mapping between the meccano boundary and the object surface must be defined. Some effort should be made in that respect in the future.

In the following section we present a description of the main stages of the new mesh generation procedure. In section 3 we show test problems and practical applications which illustrate the efficiency of this strategy. Finally, conclusions and future research are presented in section 4.

\section{Description of the Mesh Generator}

In this section, we present the main ideas that have been introduced in the mesh generation procedure. The following algorithm describes the whole mesh generation approach (for an example of this process, see Figure 5):

Mesh generation

(1) Construct a meccano approximation formed by cuboids.

(2) Define an admissible mapping between the meccano approximation and the object boundaries.

(3) Construct a valid hexahedral mesh of the meccano approximation.

(4) Construct a coarse tetrahedral mesh from the previous hexahedral mesh.

(5) Generate a local refined tetrahedral mesh of the meccano for a given precision.

(6) Move the boundary nodes of the meccano to the object surface according to the mapping defined in 2 .

(7) Relocate the inner nodes of the meccano.

(8) Optimize the actual tetrahedral mesh applying the simultaneous untangling and smoothing procedure.

In section 2.1 and 2.2, we start with the definition of the domain and its subdivision in an initial 3-D triangulation that verifies the restrictions imposed by ALBERTA. In section 2.3, we continue with the presentation of different strategies to obtain an adapted mesh which can approximate the boundaries of the domain within a given precision. We construct a mesh of the domain by projecting the boundary nodes from a meccano plane face to the true boundary surface and by relocating the inner nodes. These two steps are summarized in section 2.4 and 2.5, respectively. Finally, in section 2.6 we present a procedure to optimize the resulting mesh. 


\subsection{Object Meccano}

The first step of the procedure is to construct a meccano approximation by connecting cuboids. In general this is a non-valid hexahedral mesh. The general idea of the meccano technique could be understood as the connection of different polyhedral pieces. The use of cuboid pieces is an initial particular case.

Once the meccano approximation is fixed, we have to define an admissible mapping between the boundary faces of the meccano and the boundary of the object. We now introduce this concept. Let $\Sigma_{0}$ be the boundary of the meccano and $\Sigma$ the boundary of the object. We denote $\Sigma_{0}^{i}$ the $i$-th face of the meccano boundary, such that $\Sigma_{0}=$ $\bigcup_{i=1}^{n} \Sigma_{0}^{i}$ where $n$ is the number of meccano boundary faces. We define $\Pi: \Sigma_{0} \rightarrow \Sigma$ as a piecewise function, such that $\Pi_{\mid \Sigma_{0}^{i}}=\Pi^{i}$ where $\Pi^{i}: \Sigma_{0}^{i} \rightarrow \Pi^{i}\left(\Sigma_{0}^{i}\right) \subset \Sigma$. Then, $\Pi$ is called an admissible mapping if it satisfies:

(1) Functions $\left\{\Pi^{i}\right\}_{i=1}^{n}$ are compatible on $\Sigma_{0}$. That is $\Pi_{\mid \Sigma_{0}^{i} \cap \Sigma_{0}^{j}}^{i}=\Pi_{\mid \Sigma_{0}^{j} \cap \Sigma_{0}^{i}}^{j}, \forall i, j=$ $1, \ldots, n$, with $i \neq j$ and $\Sigma_{0}^{i} \cap \Sigma_{0}^{j} \neq \emptyset$.

(2) Global mapping $\Pi$ is continuous and biyective between $\Sigma_{0}$ and $\Sigma$.

(3) Functions $\Pi^{i}$ are differentiable on $\Sigma_{0}^{i}$.

We note that, if the mesh size of the meccano boundary is not small enough, the resulting surface mesh could be non-valid. The appropriate element size depends on the variation of the gradient of $\Pi^{i}$. We also note that admissible mapping is not unique. Obviously, the quality of the resultant surface mesh depends on the chosen mapping.

\subsection{Coarse Tetrahedral Mesh of the Meccano}

The meccano is now decomposed into a coarse and valid hexahedral mesh by an appropriate subdivision of initial cuboids. Then, we build a coarse tetrahedral mesh by splitting each hexahedron into six tetrahedra [17]. For this purpose, it is necessary to define a main diagonal on each hexahedron and corresponding diagonal on its faces. For an example of the subdivision of a cube, see Figure 1(a). In order to get a conforming tetrahedral mesh, all hexahedra are subdivided in the same way, maintaining compatibility between the diagonal of their faces. The resulting initial mesh $\tau_{1}$ can be introduced in ALBERTA since it verifies the imposed restrictions about topology and structure. The user can introduce in the code the necessary number of recursive global bisections [17] for fixing a uniform element size in the whole initial mesh. Three consecutive global bisections for a cube are presented in Figures 1 (b), (c) and (d). The resulting mesh of Figure 1(d) contains 8 cubes similar to the one shown in Figure 1(a). Obviously, the quality of the resulting tetrahedral mesh is directly related to the quality of the previous hexahedral mesh. Therefore, 


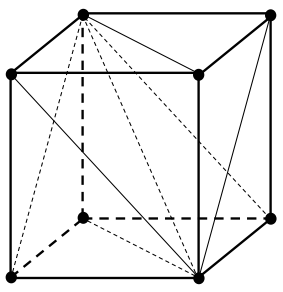

(a)

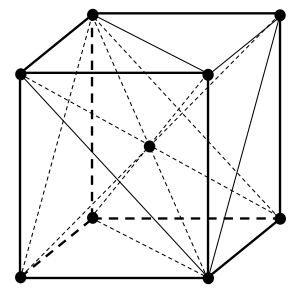

(b)

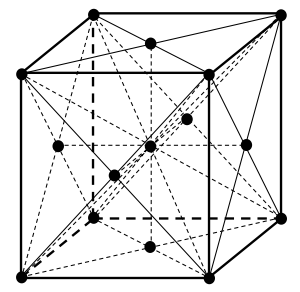

(c)

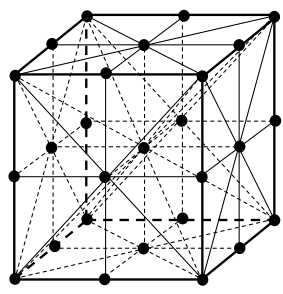

(d)

Fig. 1. Refinement of a cube by using Kossaczky's algorithm: (a) cube subdivision into six tetrahedra, (b) bisection of all tetrahedra by inserting a new node in the cube main diagonal, (c) new nodes in diagonals of cube faces and (d) global refinement with new nodes in cube edges

although the ideal case is the subdivision of the cuboids into cubes, it is not always possible.

If we consider a meccano composed of hexahedra pieces instead of cuboids, a similar technique can be applied. In this case, the recursive local refinement [17] may produce poor quality elements, depending on the initial mesh quality. The minimum quality of refined meshes is function of the initial mesh quality. A study of this aspect can be seen in $[19,32]$. In this paper, as a first approach, we have used a decomposition of the object meccano into cuboids.

\subsection{Local Refined Mesh of the Meccano}

The next step in the mesh generator includes a recursive adaptive local refinement strategy of those tetrahedra with a face placed on a boundary face of the initial coarse mesh. The refinement process is done in such a way that the true surfaces are approximated by a linear piecewise interpolation within a given precision. That is, we seek an adaptive triangulation on the meccano boundary faces, so that the resulting triangulation after node mapping on the object true boundary is a good approximation of this boundary. The user has to introduce as input data a parameter $\varepsilon$, which is a tolerance to measure the separation allowed between the linear piecewise interpolation and the true surface. At present, we have considered two criteria: the first related to the Euclidean distance between both surfaces and the second attending to the difference in terms of volume.

To illustrate these criteria, let $a b c$ be a triangle placed on the meccano boundary, and $a^{\prime} b^{\prime} c^{\prime}$ the resulting triangle after projecting the nodes $a, b$ and $c$ on surface $\Sigma$, see Figure 2. We define two different criteria to decide whether it is necessary to refine the triangle (and consequently the tetrahedra containing it) in order to improve the approximation.

For any point $Q$ in the triangle $a b c$ we define $d_{1}^{Q}$ as the euclidean distance between the mapping of $Q$ on $\Sigma, Q^{\prime}$, and the plane defined by $a^{\prime} b^{\prime} c^{\prime}$. This definition is an 


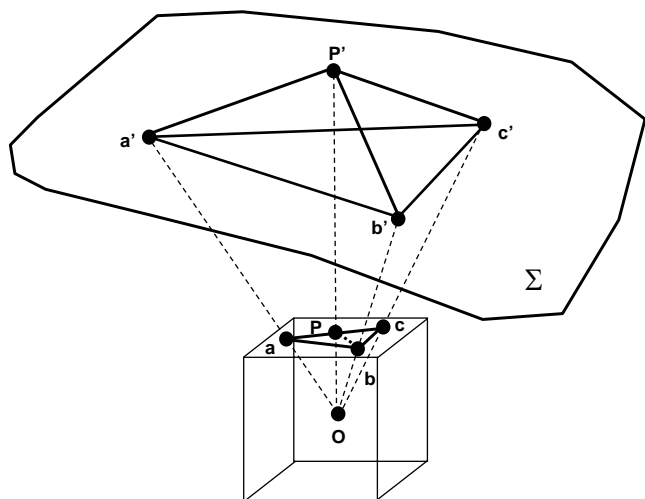

(a)

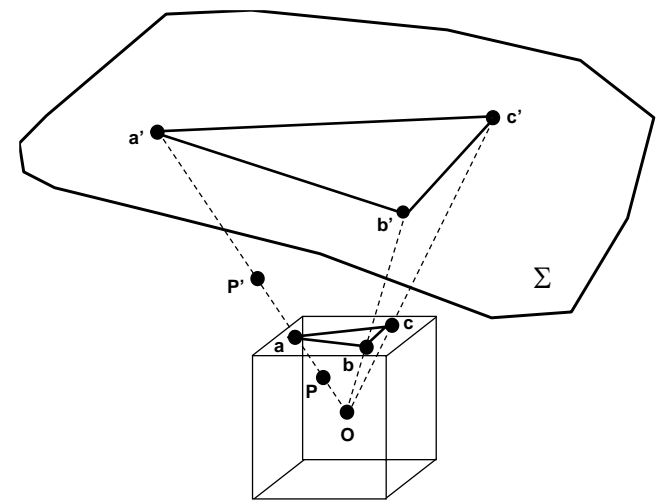

(b)

Fig. 2. Node mapping from meccano to real domain: (a) transformation of an external node $\mathrm{P}$ and $(\mathrm{b})$ of an inner node $\mathrm{P}$

estimate of the distance between the surface of the object and the current piecewise approximation.

We also introduce a measure in terms of volume, then, for any $Q$ in the triangle $a b c$ we define $d_{2}^{Q}$ as the volume of the virtual tetrahedron $a^{\prime} b^{\prime} c^{\prime} Q^{\prime}$. In this case, $d_{2}^{Q}$ is an estimate of the lost volume in the linear approximation by the face $a^{\prime} b^{\prime} c^{\prime}$ of the true surface.

The threshold of whether to refine the triangle or not is given by a tolerance $\varepsilon_{i}$ fixed by the user. With the previous definition, $d_{1}^{Q}<\varepsilon_{1}$ for all $Q$ in the boundary on the meccano implies that the distance between the surface of the object and its piecewise linear approximation is less than $\varepsilon_{1}$. On the other hand, $d_{2}^{Q}<\varepsilon_{2}$ for all $Q$ in the boundary on the meccano would mean that the lost volume per boundary face is bounded by $\varepsilon_{2}$. Alternatively, $\varepsilon_{2}$ could be defined as the allowed difference of volumes and we could use an equidistribution strategy as is usual in a-posteriori error estimates. Nevertheless, here we prefer to use here a local version of $\varepsilon_{2}$, so the difference of volumes is estimated by multiplying $\varepsilon_{2}$ by the number of boundary faces of the final approximation.

Obviously, other measures could be introduced in line with the desired approximation type (curvature, points properties, etc.). What is more, the user could consider the combination of several measures simultaneously.

Once we have defined separation measures $d_{i}$ and the corresponding tolerances $\varepsilon_{i}$, we propose two different strategies for reaching our objective in the following subsections. 


\subsubsection{Sequence of Refinements and Derefinement}

The first strategy consists of a simple method. It combines a sequence of refinement steps with one derefinement step.

Simple refinement step. We construct a sequence of tetrahedral nested meshes by recursive bisection of all tetrahedra that contain a face located on the meccano boundary faces; see Figure 1 . The number of bisections $n_{b}$ is determined by the user as a function of the desired resolution. At this point, we identify the true surface with the linear approximation obtained with this resolution. So, we have a uniform distribution of nodes on these meccano faces and we can consider their virtual mapping on the object boundary.

Derefinement step. We apply a derefinement procedure, which is a generalization of the strategy developed in [8]. This criterion fixes which tetrahedra introduced in the refinement sequence can be eliminated without damaging the approximation for the prescribed tolerance $\varepsilon_{i}$. The derefinement is applied iteratively to the current mesh and concludes either when there are no elements to remove or when the coarse mesh is reached.

Note that each tetrahedron $T$ (generated by bisection of its father) has a so-called newest node $P$. This node was introduced at the middle point of a prescribed edge $a c$ of the father of $T$ to generate its two sons, see Figure 2(a). The derefinement criterion is efficient because it only computes the distance $d_{i}^{P}$ relative to this newest node to decide if the tetrahedron $T$ could be removed. This distance is related to the face $a b c$ (or its virtual mapping $a^{\prime} b^{\prime} c^{\prime}$ ) of the father of $T$.

Then, the derefinement criterion, associated to a tetrahedron $T$ of the sequence of nested meshes, can be introduced as:

Derefinement criterion. Tetrahedron $T$ is marked to be derefined, if it satisfies one of the following conditions:

(1) The newest node $P$ of $T$ is interior.

(2) The newest node $P$ of $T$ is placed on the boundary of the meccano and $d_{i}^{P}<\varepsilon_{i}$.

A marked tetrahedron $T$ will be removed only if all the elements generated by the bisection of the edge $a c$ of its father are also marked. Finally, the refinement/derefinement procedure to construct a local refined tetrahedral mesh of the meccano is summarized in the following algorithm:

Refinement/derefinement procedure

(1) Set $n_{b}$ and $\varepsilon_{i}$.

(2) Construct the coarse tetrahedral mesh of the meccano.

(3) Refine $n_{b}$ times all tetrahedra with at least one face placed on the meccano boundary. 
(4) Mark for derefinement all tetrahedra that satisfies the derefinement criterion for a distance $d_{i}$ and a tolerance $\varepsilon_{i}$.

(5) Derefine the mesh.

(6) If the mesh was modified, go to step 4.

\subsubsection{Sequence of Local Refinements}

The second strategy also starts with the coarse mesh of the meccano, but it only applies local refinement to obtain the fine one. In this case the refinement criterion for tetrahedron $T$ is:

Refinement criterion. Tetrahedron $T$ is marked to be refined, if it satisfies the following two conditions:

(1) $T$ has a face $F$ on the boundary of the meccano.

(2) $d_{i}^{Q} \geq \varepsilon_{i}$ for some point $Q$ located on face $F$ of $T$.

From a numerical point of view, the number of points $Q$ (analyzed in this strategy) is reduced to a set of points on a uniform mesh of a given resolution, or a set of points of quadrature. Finally, the refinement strategy for constructing a local refined tetrahedral mesh of the meccano is summarized in the following algorithm:

Refinement procedure

(1) Set $n_{b}$ and $\varepsilon_{i}$.

(2) Construct the coarse tetrahedral mesh of the meccano.

(3) Mark for refinement all tetrahedra which satisfies the refinement criterion for a distance $d_{i}$ and a tolerance $\varepsilon_{i}$.

(4) Refine the mesh.

(5) If the number of refinement steps is less than $n_{b}$ and the mesh was modified, go to step 3 .

While first strategy is simpler, it could lead to problems with memory requirements if the number of tetrahedra is very high before applying the derefinement algorithm. For example, this situation can occur when there are surfaces defined by very high resolution functions. Nevertheless, the user could control the number of recursive bisections $n_{b}$ and the tolerance $\varepsilon_{i}$.

On the other hand, the problem of the second strategy is to determine whether a face placed on meccano boundary must be subdivided to achieve the desired approximation of the true surface. This analysis must be done every time that a boundary face is subdivided into its two son faces. Suppose, for example, that the true surface is given by a discrete function. Then, the subdivision criterion should stop for a particular face when all the surface discretization points, defined on this face, have been analyzed and all of them verify the approximation criterion. So, this second strategy has the inconvenience that each surface discretization point could be studied many times and, therefore, it generally involves a higher computational 
cost than the first strategy. Nevertheless, both of those strategies could be faster depending on the geometry of the object surface and the parameters fixed by the user.

\subsection{External Node Mapping on Object Boundary}

Although ALBERTA has already implemented a node projection on a given boundary surface during the bisection process, it has two important restrictions: nodes belonging to the initial mesh are not projected and inverted elements could appear in the case of projecting new nodes on complex surfaces (i.e. non-convex object). In the latter case, the code does not work properly since it is only prepared to manage valid meshes.

Therefore, a new strategy must be developed in the mesh generator. The projection (or mapping) is really done once we have defined the local refined mesh by using one of the two methods proposed in the previous section. Then, the nodes placed on the meccano faces are projected (or mapped) on their corresponding true surfaces, maintaining the position of the inner nodes of the meccano triangulation. We have remarked that any one-to-one projection can be defined: orthogonal, spherical, cylindrical, etc. For example, spherical projection from point $O$ has been used in Figure 2.

After this process, we obtain a valid triangulation of the domain boundary, but a tangled tetrahedral mesh could appear. Inner nodes of the meccano could now be located even outside the domain. Thus, an optimization of the mesh is necessary. Although the final optimized mesh does not depend on the initial position of the inner nodes, it is better for the optimization algorithm to start from a mesh with as good a quality as possible. Therefore, we propose to relocate the inner nodes of the meccano in a reasonable position before the mesh optimization.

\subsection{Relocation of Inner Nodes}

There would be several strategies for defining an appropriate position for each inner node of the domain. An acceptable procedure is to modify their relative position as a function of the distance between boundary surfaces before and after their projections. This relocation is done attending to proportional criteria along the corresponding projection line. For example, relocation of inner node $P$ in its new position $P^{\prime}$, such that $O P^{\prime}=O P \times O a^{\prime} / O a$, is represented in Figure 2(b).

Although this node movement does not solve the tangle mesh problem, it normally lessens it. In other words, the resulting number of inverted elements is lower and the mean quality of valid elements is greater. 


\subsection{Object Mesh Optimization: Untangling and Smoothing}

An efficient procedure is necessary to optimize the current mesh. This process must be able to smooth and untangle the mesh and is crucial in the proposed mesh generator.

The most usual techniques to improve the quality of a valid mesh, that is, a mesh with no inverted elements, are based upon local smoothing. In short, these techniques consist of finding the new positions that the mesh nodes must hold, in such a way that they optimize an objective function. Such a function is based on a certain measurement of the quality of the local submesh, $N(v)$, formed by the set of elements connected to the free node $v$ whose coordinates are given by $\mathbf{x}$. We have considered the following objective function (1) derived from an algebraic mesh quality metric studied in [16], but it would also be possible to use other objective functions that have barriers like those presented in [15].

$$
K(\mathbf{x})=\left[\sum_{m=1}^{M}\left(\frac{1}{q_{\eta_{m}}}\right)^{p}(\mathbf{x})\right]^{\frac{1}{p}}
$$

where $M$ is the number of elements in $N(v), q_{\eta_{m}}$ is an algebraic quality measure of the $m$-th element of $N(v)$ and $\mathrm{p}$ is usually chosen as 1 or 2 . Specifically, we have considered the mean ratio quality measure, which for a tetrahedron is $q_{\eta}=\frac{3 \sigma^{\frac{2}{3}}}{|S|^{2}}$ and for a triangle is $q_{\eta}=\frac{2 \sigma}{|S|^{2}}$, being $|S|$ the Frobenius norm of matrix $S$ associated to the affine map from the ideal element (usually equilateral tetrahedron or triangle) to the physical one, and $\sigma=\operatorname{det}(S)$. Other algebraic quality measures can be used as, for example, the metrics based on the condition number of matrix $S, q_{\kappa}=\frac{\rho}{|S|\left|S^{-1}\right|}$, where $\rho=2$ for triangles and $\rho=3$ for tetrahedra.

As it is a local optimization process, we can not guarantee that the final mesh is globally optimum. Nevertheless, after repeating this process several times for all the nodes of the current mesh, quite satisfactory results can be achieved. Objective functions are usually appropriate to improve the quality of a valid mesh, but they do not work properly when there are inverted elements. This is because they present singularities (barriers) when any tetrahedron of $N(v)$ changes the sign of its Jacobian determinant. To avoid this problem it is possible to proceed as Freitag et al. in $[10,9]$, where an optimization method consisting of two stages is proposed. In the first, the possible inverted elements are untangled by an algorithm that maximizes their negative Jacobian determinants [10] while, in the second, the resulting mesh from the first stage is smoothed using another objective function based on a quality metric of the tetrahedra of $N(v)$ [9]. After the untangling procedure, the mesh has a very poor quality because the technique has no motivation to create good-quality elements. As remarked in [9], it is not possible to apply a gradient-based algorithm 
to optimize the objective function because it is not continuous all over $\mathbb{R}^{3}$, making it necessary to use other non-standard approaches.

We have proposed an alternative to this procedure [7], so the untangling and smoothing are carried out in the same stage. For this purpose, we use a suitable modification of the objective function such that it is regular all over $\mathbb{R}^{3}$. It consists of substituting the the term $\sigma$ in the quality metrics with the positive and increasing function $h(\sigma)=\frac{1}{2}\left(\sigma+\sqrt{\sigma^{2}+4 \delta^{2}}\right)$. When a feasible region (subset of $\mathbb{R}^{3}$ where $v$ could be placed, $N(v)$ being a valid submesh) exists, the minima of the original and modified objective functions are very close and, when this region does not exist, the minimum of the modified objective function is located in such a way that it tends to untangle $N(v)$. The latter occurs, for example, when the fixed boundary of $N(v)$ is tangled. With this approach, we can use any standard and efficient unconstrained optimization method [1] to find the minimum of the modified objective function.

In addition, a smoothing of the boundary surface triangulation could be applied before the movement of inner nodes of the domain by using the new procedure presented in [6] and [22]. This surface triangulation smoothing technique is also based on a vertex repositioning defined by the minimization of a suitable objective function. The original problem on the surface is transformed into a two-dimensional one on the parametric space. In our case, the parametric space is a plane, chosen in terms of the local mesh, in such a way that this mesh can be optimally projected performing a valid mesh, that is, without inverted elements.

\section{Test Examples}

The performance of our new mesh generator is shown in the following applications. The first corresponds to a domain defined over a complex terrain, the second to a torus, the third to an object with two different materials and the fourth to a 3-D scanned object whose boundary is defined with a surface triangulation. For all presented examples, the meccano cuboids are subdivided into a valid mesh of cubes.

\subsection{Domain over Complex Terrain}

In the last few years, we have developed a tetrahedral mesh generator that approximates the orography of complex terrains with a given precision $[23,24]$. To do so, we only have digital terrain information. Our domain is limited on its lower part by the terrain and on its upper part by a horizontal plane placed at a height at which the magnitudes under study may be considered steady. The lateral walls are formed by 
four vertical planes. The generated mesh could be used for numerical simulation of environmental phenomena, such as wind field adjustment [26], fire propagation or atmospheric pollution [25]. The following procedures were mainly involved in this former automatic mesh generator: a Delaunay triangulation method [5,13], a 2-D refinement/derefinement algorithm [8] and a simultaneous untangling and smoothing algorithm [7]. Moreover, we have recently developed a new method for improving the quality of surface triangulations by using optimal local projections $[6,22]$, which can be introduced in the mesh generator.

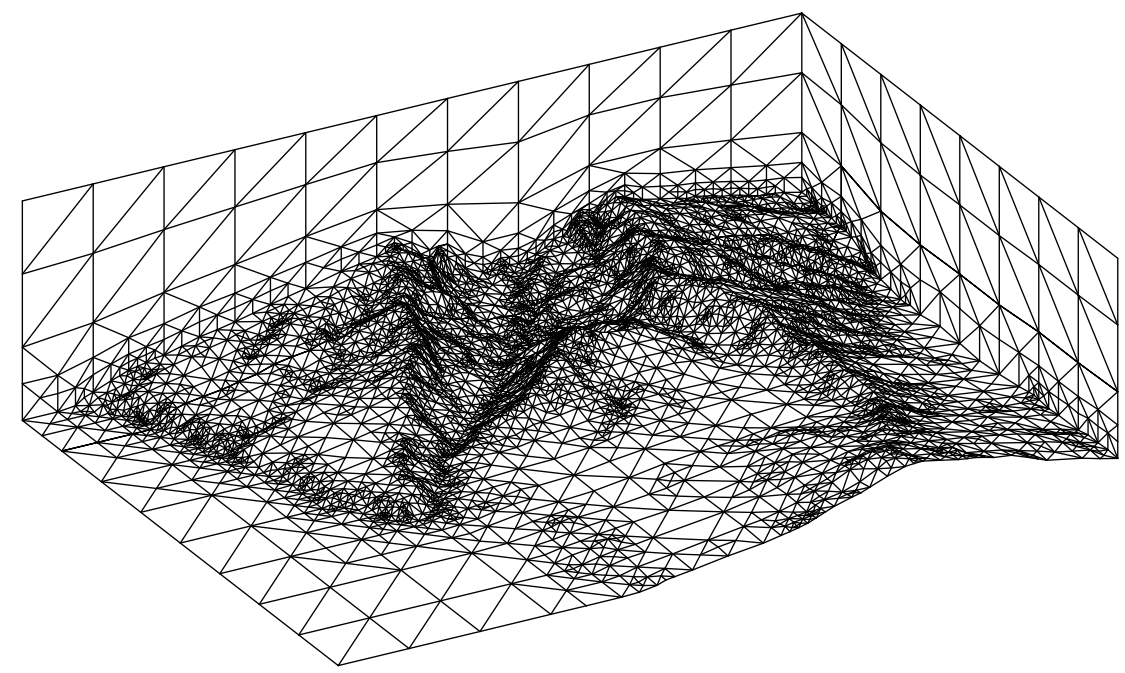

(a)

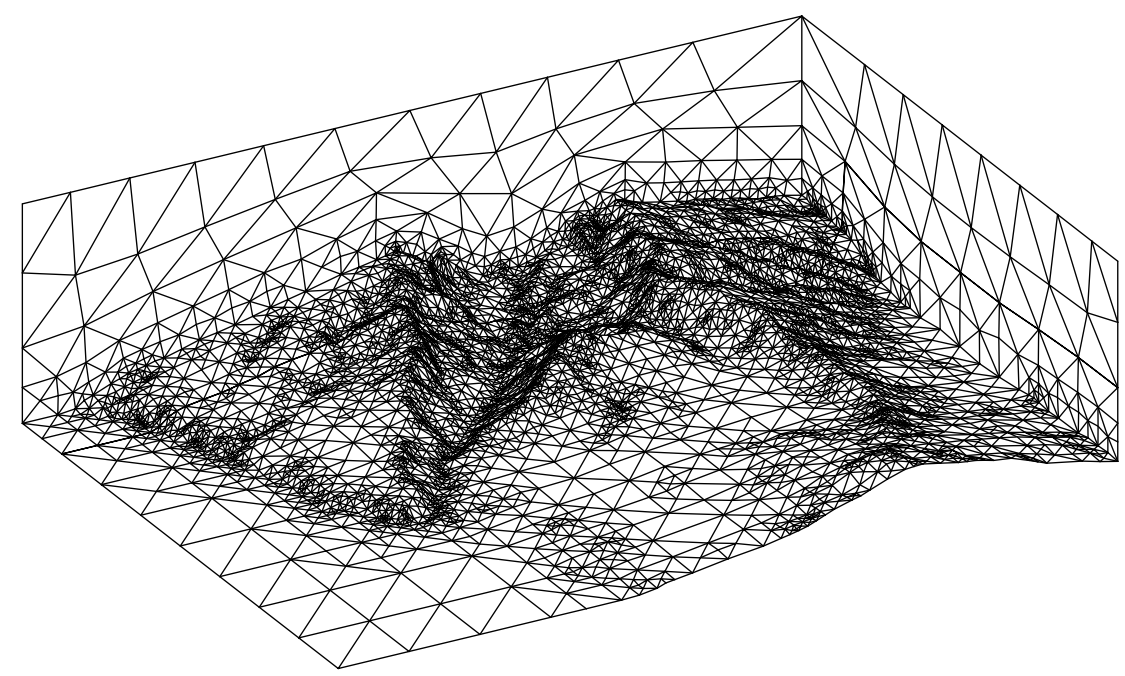

(b)

Fig. 3. Detail of Isla de La Palma (Canary Island): (a) initial mesh and (b) resulting mesh after five steps of the optimization process

As an alternative to this strategy, the new automatic mesh generator proposed in this paper can be used for the same purpose. As a practical application we have 
considered a rectangular area in Isla de La Palma (Canary Islands) of $22 \times 16 \mathrm{~km}$. The upper boundary of the domain has been placed at $h=6 \mathrm{~km}$. To define the topography we use a digitalization of the area where heights are defined over a uniform grid with a spacing step of $200 \mathrm{~m}$ in directions $x$ and $y$. We start from a cuboid (meccano) of $22 \times 16 \times 6 \mathrm{~km}$ initially subdivided into $11 \times 8 \times 3$ cubes with edge sizes of $2 \mathrm{~km}$. Each cube is subdivided into six tetrahedra by using the subdivision proposed in [17], see Figure 1(a). This discretization is used to define the uniform initial triangulation $\tau_{1}$ of the parallelepiped. We refine it 18 times by constructing a recursive bisection of all tetrahedra containing a face placed on the lower face of the parallelepiped. If we applied 6 global refinements by using the 4-T Rivara's algorithm [28] instead of previous recursive bisections, the resultant 2-D triangulation on the lower face of the parallelepiped would be the same.

Once the orography is virtually interpolated on this local refined mesh, the derefinement condition is applied with a derefinement parameter related to distance $\varepsilon_{1}=25$ $m$. Then, we make an orthogonal projection on the terrain of the adaptive triangulation obtained on the lower face of the parallelepiped. In addition, we relocate the other nodes vertically by using a proportional criterion. The adapted mesh has 65370 tetrahedra and 15263 nodes, see Figure 3(a), and it nears the terrain surface with an error less than $\varepsilon_{1}=25 \mathrm{~m}$.

This mesh has 115 inverted tetrahedra, its average quality measure is $\bar{q}_{\kappa}=0.68$ and its minimum quality is 0.091, see reference [7] and Figure 4. The node distribution is hardly modified after five steps of the optimization process by using our modified objective function. We remark that, during this optimization process, we have not relocated those nodes placed on the terrain.

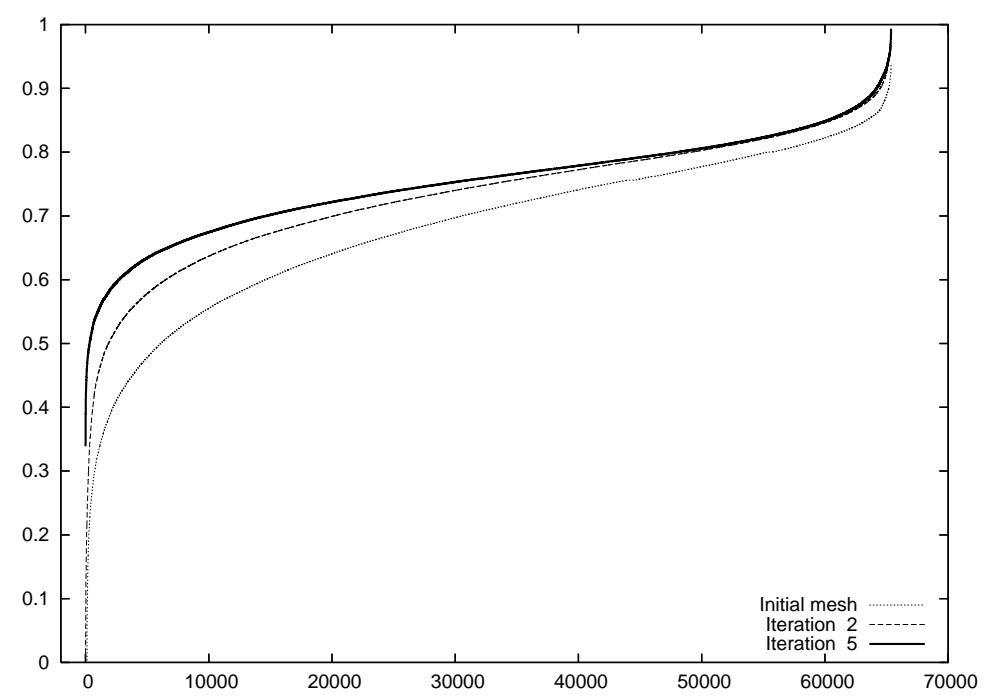

Fig. 4. Quality curves, using $q_{\kappa}=\frac{3}{|S| \mid S^{-1}}$, for the initial and optimized meshes after two and five iterations for the domain defined in Isla de La Palma (Canary Island)

The evolution of the mesh quality during the optimization process is represented in 
Figure 4. These curves are obtained by sorting the elements in increasing order of quality. This measure tends to stagnate quickly. The quality curves corresponding to the second and fifth optimization steps are close. The average quality measure increases to $\bar{q}_{\kappa}=0.75$. After this optimization process, the worst quality measure of the optimized mesh tetrahedra is 0.34 . Finally, we remark that the number of parameters necessary to define the resulting mesh is quite low, as is the computational cost. The total CPU time for the initial mesh and its optimization is less than 1 minute on an Intel Pentium M processor, $2.26 \mathrm{GHz}$ and $2 \mathrm{~Gb}$ RAM memory. In particular, the computational cost of five iterations of the simultaneous untangling and smoothing procedure is about half a minute. At the first iteration of this optimization process the mesh is untangled.

\subsection{Torus}

We now consider the automatic 3-D triangulation of a torus. We start from a simple meccano composed of four cuboids as shown in Figure 5(a). This meccano is included in a parallelepiped whose dimensions are $6 \times 6 \times 2$ and which contains the directrix circumference of the torus. Therefore, the definition of a one-to-one projection between the meccano and torus boundaries is straightforward.

In the first step of the mesh generator procedure, the meccano is splitted into a coarse and uniform valid mesh with 64 cubes, see Figure 5(b). As each cube is divided into six tetrahedra, it results in an initial 3-D meccano triangulation of 384 tetrahedra, see Figure 5(c). In the following step, we apply 9 recursive bisections on all tetrahedra which have a face placed on the meccano boundary. This mesh contains 15364 nodes and 61328 tetrahedra. Then, we virtually project meccano boundary nodes on the torus boundary. Attending to this virtual projection, the refinement/derefinemet algorithm is applied with a derefinement parameter, related to volume, $\varepsilon_{2}=0.0001$. The resulting adaptive mesh contains 2032 nodes and 7840 tetrahedra and it is shown in Figure 5(d). The real projection of this meccano surface triangulation on the torus surface produces a 3-D tangled mesh with 1424 inverted elements, see Figure 5(e). The relocation of inner nodes by using a proportional criterion reduces the number of inverted tetrahedra to 240 . The average quality measure of this tangled mesh is $\bar{q}_{\kappa}=0.63$. If we use the tetrahedral mesh optimization presented in [7], the mesh quality is improved to a minimum value of 0.39 and an average $\bar{q}_{\kappa}=0.73$ after two iterations. The evolution of the mesh quality during the optimization process to the inner nodes of the domain, is represented in Figure 6. The quality curves of following iterations are very close. The final mesh can be seen in Figure 5(f).

We remark that, due to the high quality surface triangulation obtained with our method, the mesh improvement is not significant if we previously apply the smoothing surface triangulation algorithm introduced in [6]. The CPU time for construct- 


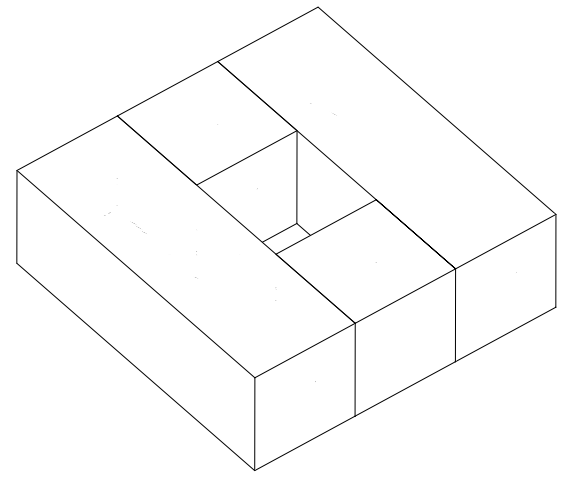

(a)

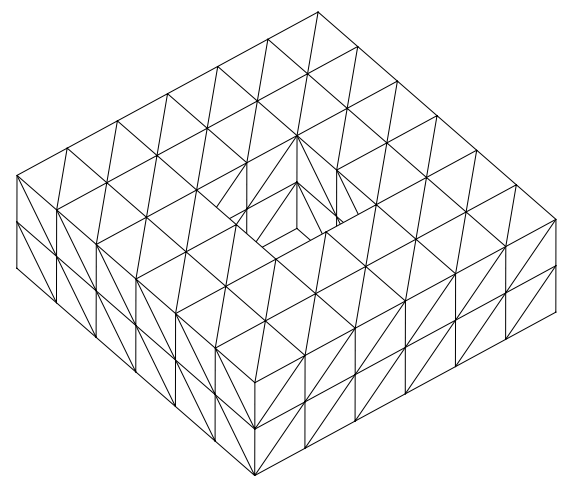

(c)

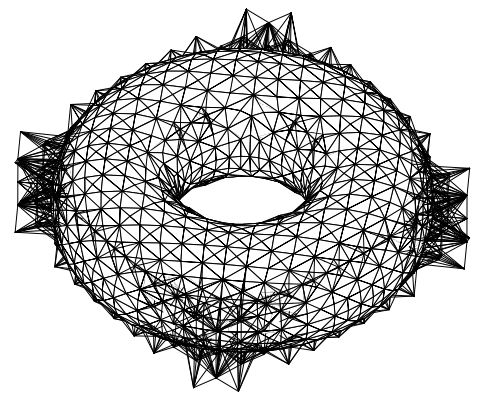

(e)

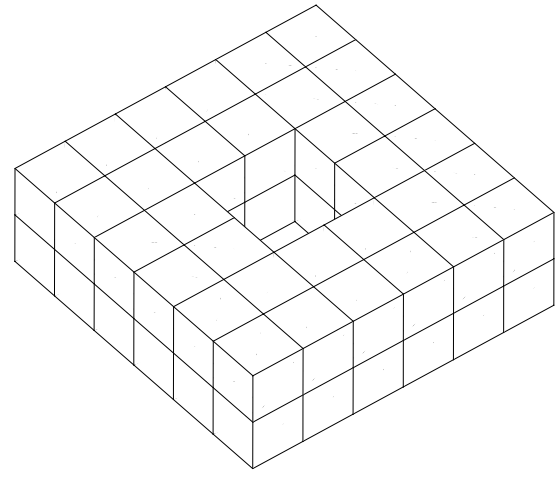

(b)

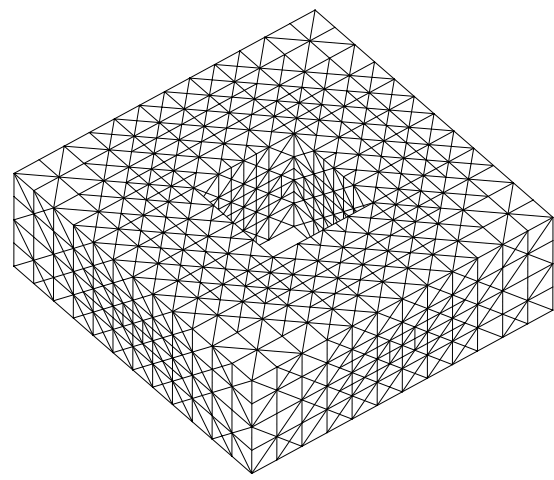

(d)

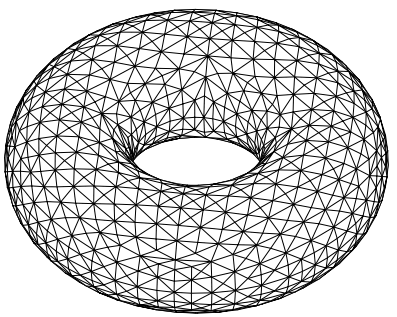

(f)

Fig. 5. Main stages of the mesh generator for the torus: (a) meccano approximation formed by four cuboids, (b) valid mesh of cubes, (c) coarse tetrahedral mesh, (d) mesh adaption after applying the refinement/derefinement procedure, (e) tangled mesh after the projection on torus surface and (f) resulting mesh after inner node relocation and mesh optimization

ing the initial mesh is approximately 0.5 seconds and for its optimization process is 2.5 seconds on an Intel Xeon processor, $3.06 \mathrm{GHz}$ and $4 \mathrm{~Gb}$ RAM memory. 


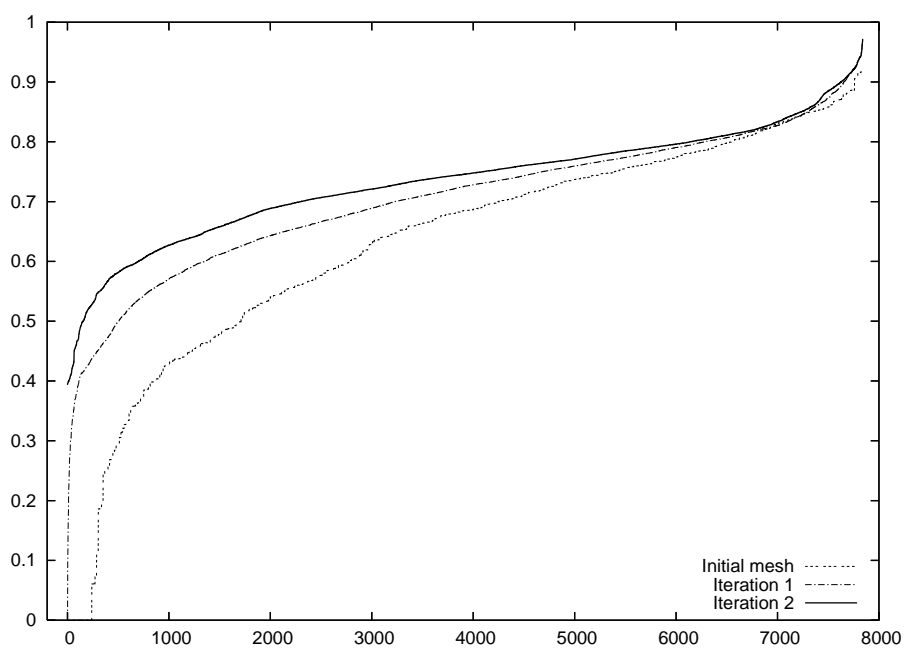

Fig. 6. Quality curves, using $q_{\kappa}=\frac{3}{|S|\left|S^{-1}\right|}$, for the initial and optimized meshes after two iterations for the torus

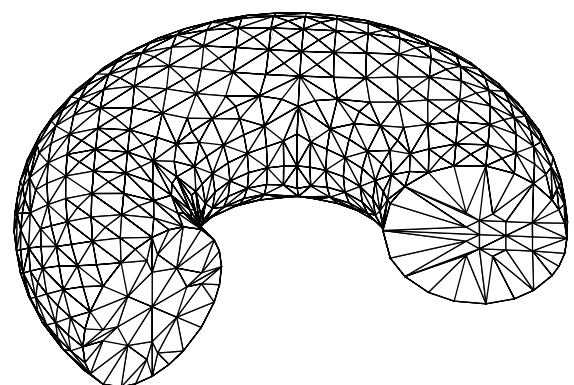

(a)

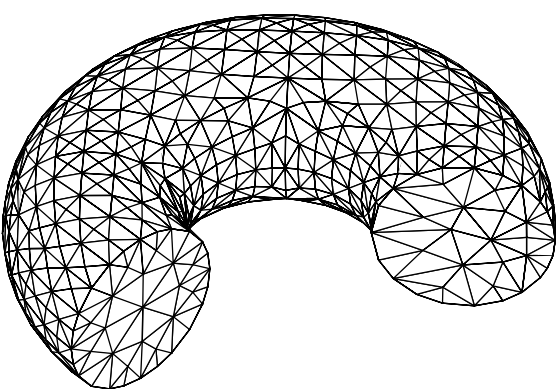

(b)

Fig. 7. Cross sections of the torus before (a) and after (b) the application of the mesh optimization process

In order to show the efficiency of the mesh optimization technique inside the torus after relocation of inner nodes, we display in Figure 7 two cross sections before (a) and after (b) its application. We note that the optimization procedure can lead to the final mesh without any relocation of inner nodes. Obviously, the necessary number of iterations and CPU time to obtain a similar final mesh quality increases in this case. The CPU time for optimization procedure (four untangling iterations and five smoothing iterations) should be 3.8 seconds instead of 2.5 seconds.

Finally we analyze the relation between the derefinement parameter $\varepsilon_{2}$ and the surface deviation. We report in Table 1 for several values of $\varepsilon_{2}$ : the number of boundary faces of the resulting mesh (\#Faces), the volume of the piecewise linear approximation $\left|\Omega_{k}\right|$, the relative difference of volumes between the true object and the piecewise linear approximation $\left(\frac{|\Omega|-\left|\Omega_{k}\right|}{|\Omega|}\right)$, the volume of the set-theoretic difference of $\Omega$ and $\Omega_{k}$, an estimation of this value (\#Faces $\times \varepsilon_{2}$ ), and the ratio between the estimation and true difference. The results show that $\varepsilon_{2}$ correctly controls the 


\begin{tabular}{|c|c|c|c|c|c|c|}
\hline$\varepsilon_{2}$ & \#Faces & $\left|\Omega_{k}\right|$ & $\frac{|\Omega|-\left|\Omega_{k}\right|}{\Omega \Omega \mid}(\%)$ & $\left|\Omega_{k} \backslash \Omega \cup \Omega \backslash \Omega_{k}\right|$ & \#Faces $\times \varepsilon_{2}$ & $\frac{\text { \#Faces } \times \varepsilon_{2}}{\left|\Omega_{k} \backslash \Omega \cup \Omega \backslash \Omega_{k}\right|}$ \\
\hline $10^{-4}$ & 2336 & 39.0842 & $0.99 \%$ & 0.4316 & 0.2336 & 1.84 \\
\hline $10^{-5}$ & 8016 & 39.3655 & $0.28 \%$ & 0.1269 & 0.0801 & 1.58 \\
\hline $10^{-6}$ & 24416 & 39.4421 & $0.09 \%$ & 0.0423 & 0.0244 & 1.72 \\
\hline $10^{-7}$ & 74048 & 39.4668 & $0.02 \%$ & 0.0137 & 0.0074 & 1.84 \\
\hline $10^{-8}$ & 241216 & 39.4747 & $0.01 \%$ & 0.0042 & 0.0024 & 1.76 \\
\hline
\end{tabular}

Table 1

Relation between the derefinement parameter $\varepsilon_{2}$ and the surface deviation. The volume of the true torus is $4 \pi^{2} \approx 39.4784$

derefinement procedure and that $\varepsilon_{2} \times \#$ Faces is a good and cheap estimation of the quality of the approximation obtained.

\subsection{Object with Two Different Materials}

In order to show the efficiency of our automatic tetrahedral mesh generator on more complex domains, we now consider the discretization of a glass with liquid. The input data consists on a meccano of five cuboids for the glass and one cuboid for the liquid, see Figure 8(a). This meccano is included in a parallelepiped whose dimensions are $5 \times 5 \times 8$. The new mesh generation strategy automatically defines the boundary between the two materials and achieves a good mesh adaption to the geometrical domain characteristics. A one-to-one projection between meccano and object is defined by a cylindrical projection for vertical faces and an orthogonal one for horizontal faces.

The meccano is splitted into a 3-D triangulation of 1146 tetrahedra and 320 nodes. We apply 9 recursive bisections on all tetrahedra which have a face placed on the meccano boundary or on the material interface. This mesh contains 42811 nodes and 195272 tetrahedra. The derefinement parameter is $\varepsilon_{2}=0.0002$ in this case. The resulting adaptive mesh contains 2722 nodes and 12520 tetrahedra and is shown in Figure 8(b). The projection of this meccano surface triangulation on the true surface produces a 3-D tangled mesh with 4192 inverted elements, see Figure 8(c). In order to highlight the capability of the optimization procedure [7], in this case, we do not relocate any inner node. After nine iterations, this mesh optimization algorithm converts the tangled mesh into the one presented in Figure 8(d). The mesh quality is improved to a minimum value of 0.21 and an average $\bar{q}_{\kappa}=0.51$. The quality curves for the initial and final triangulations are shown in Figure 9. The CPU time for constructing the initial mesh is approximately 2 seconds and for its optimization is 12 seconds on a Intel Xeon processor, $3.06 \mathrm{GHz}$ and $4 \mathrm{~Gb}$ RAM memory.

To reflect the difficulty of this test problem, we present two object cross sections in Figure 10. In the first, we plot the glass (a) and in the second, we include the liquid (b). It is interesting to observe the mesh conformity with the material interface. Moreover, the mesh generator constructs an appropriate discretization of the thin 
glass walls.

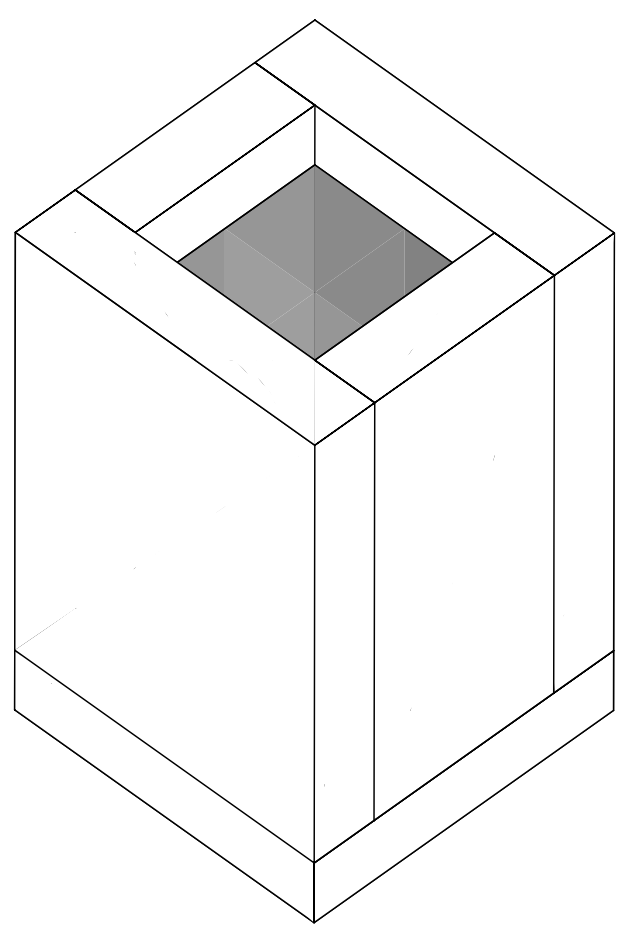

(a)

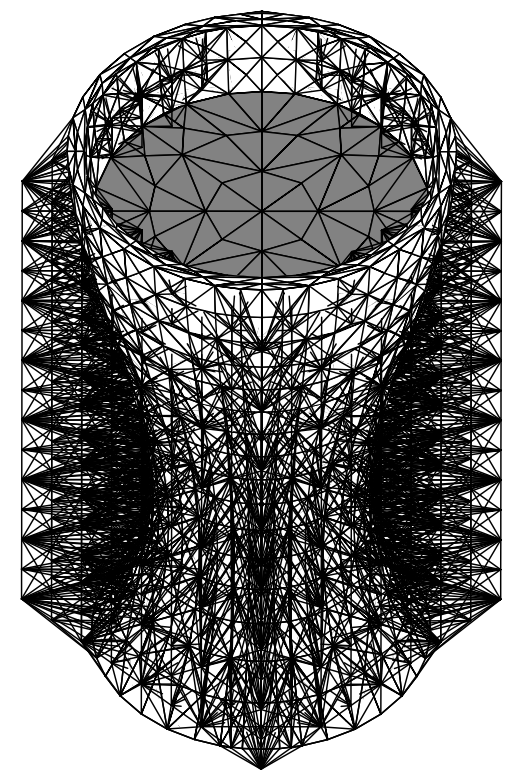

(c)

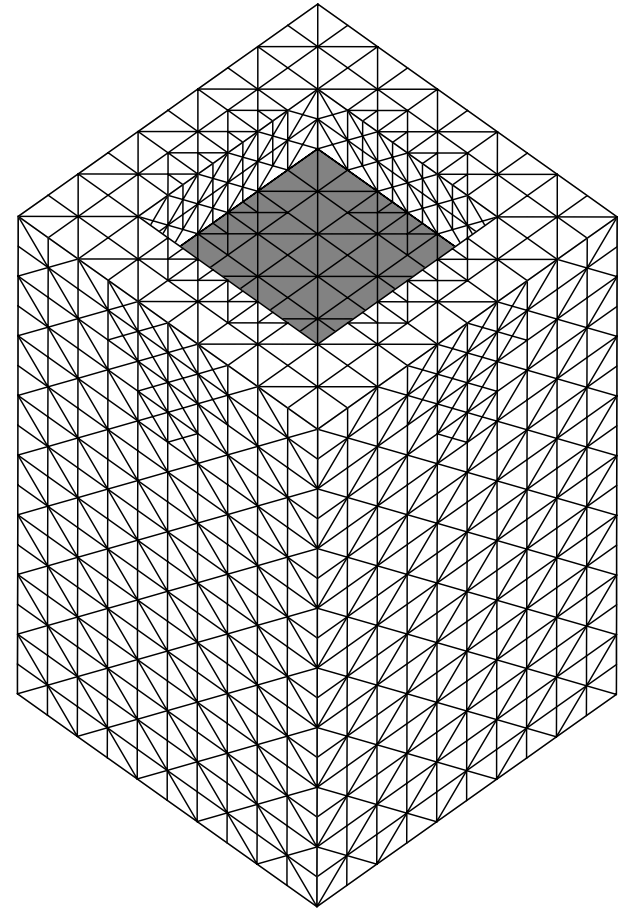

(b)

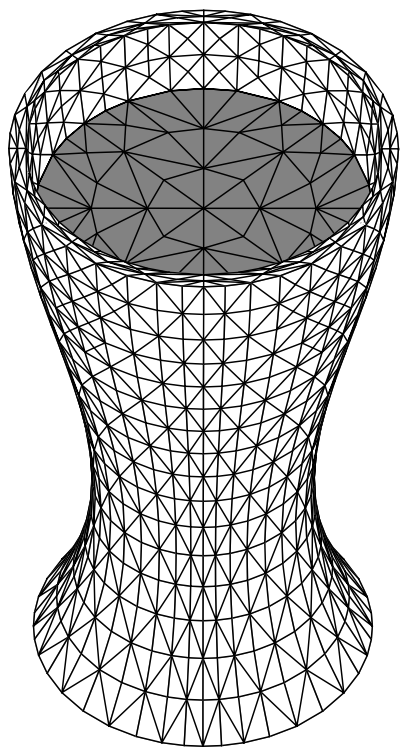

(d)

Fig. 8. Main stages of the mesh generator for the glass with liquid: (a) meccano, (b) mesh adaption after applying the refinement/derefinement procedure, (c) tangled mesh after the projection on torus surface and (d) resulting optimized mesh 


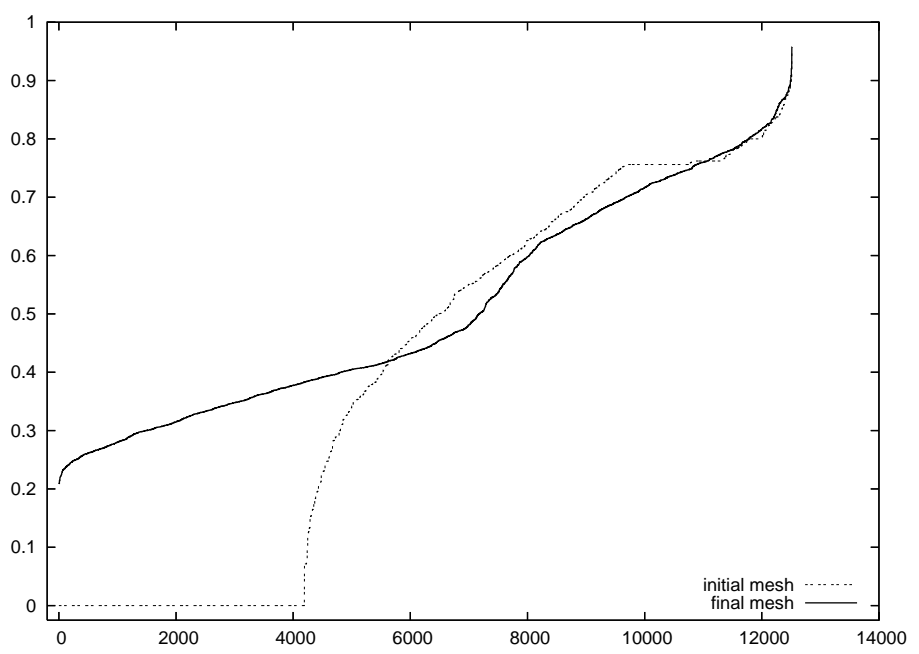

Fig. 9. Quality curves, using $q_{\kappa}=\frac{3}{|S|\left|S^{-1}\right|}$, for the initial tangled mesh and the optimized triangulation after nine iterations for the glass with liquid

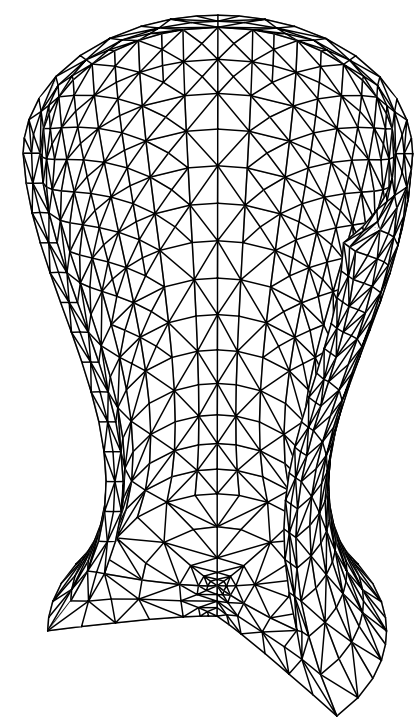

(a)

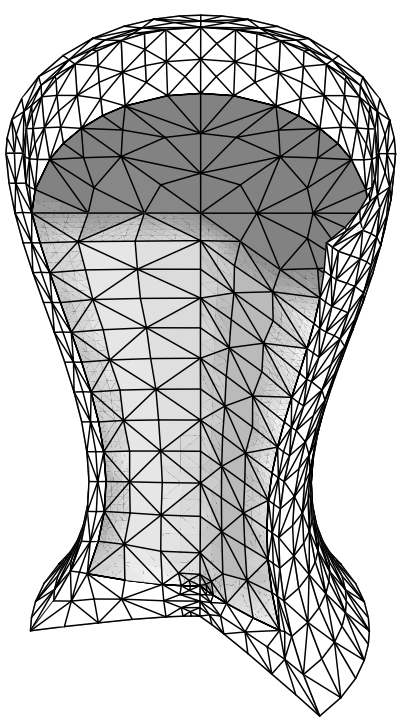

(b)

Fig. 10. Cross sections of the glass without (a) and with (b) liquid

\subsection{Scanned Object}

In this last example, we have applied our technique to construct a 3-D triangulation of Igea. A surface triangulation of this object is fixed by using a 3-D scanner and it can be obtained from $h t t p: / / w w w . c y b e r w a r e . c o m /$. We use this information to define the object surface. The original surface triangulation of Igea has 67170 triangles and 33587 nodes, see Figure 11(a). Note the poor quality of this original mesh in several parts. The initial value of the average quality for this surface triangulation 
is 0.79 (measured with the quality metric based on the condition number [9]) and the minimum is 0.10 .

The 3-D mesh is automatically constructed starting from only one $60 \times 60 \times 60$ cube and using a spherical projection with a derefinement parameter $\varepsilon_{2}=0.005$. We choose the focus point close to the centroid of the surface nodes. The surface triangulation of the resulting 3-D mesh is shown in Figure 11(b) with 39012 triangles and 19508 nodes. Its average quality increases to 0.84 and its minimum to 0.16. We have compared the initial volume of Igea, see Figure 11(a), and of the final surface triangulation, Figure 11(b), resulting in 278478 and 278389, respectively. This means a $0.03 \%$ decrease in the volume that is directly related with $\varepsilon_{2}$.

In order to show the efficiency of mesh adaption in the interior of Igea, we present in Figure 12 a cross section of the 3-D mesh before (a) and after optimization (b). It is interesting to observe the inner mesh adaption in function of the surface geometry. In this case, the 3-D mesh generator constructs a discretization of Igea with 174952 tetrahedra and 41215 nodes. The mesh quality is improved to a minimum value of 0.14 and its average quality increases from $\bar{q}_{\kappa}=0.28$ to $\bar{q}_{\kappa}=0.70$. The CPU time for constructing the initial mesh is approximately 15 seconds and for its optimization is 56 seconds on a Intel Xeon processor, $3.06 \mathrm{GHz}$ and $4 \mathrm{~Gb}$ RAM memory.

Finally, we remark again that, due to the high quality surface triangulation obtained with our method, application of the smoothing surface triangulation algorithm [6] does not produce significant improvements. The resulting average and minimum qualities are 0.85 and 0.18 , respectively.

\section{Conclusions and Future Research}

The proposed mesh generator is an efficient method for creating tetrahedral meshes on domains with boundary faces projectable on a meccano boundary. We remark that it requires minimum user intervention and has a low computational cost. The main ideas presented in this paper for automatic mesh generation, which have been implemented in ALBERTA, could be used for different codes which work with other tetrahedral or hexahedral local refinement/derefinement algorithms. Taking these ideas into account, complex domains could be meshed by decomposing its outline into a set of connected cuboids. In future works, new types of pieces and connections could be considered for constructing the meccano.

Although this procedure is at present limited in applicability for highly complex geometries, it results in a very efficient approach for the problems that fall within the mentioned class. At present, the user has to define the meccano associated to the object and mapping between meccano and object surfaces. Once these aspects are 


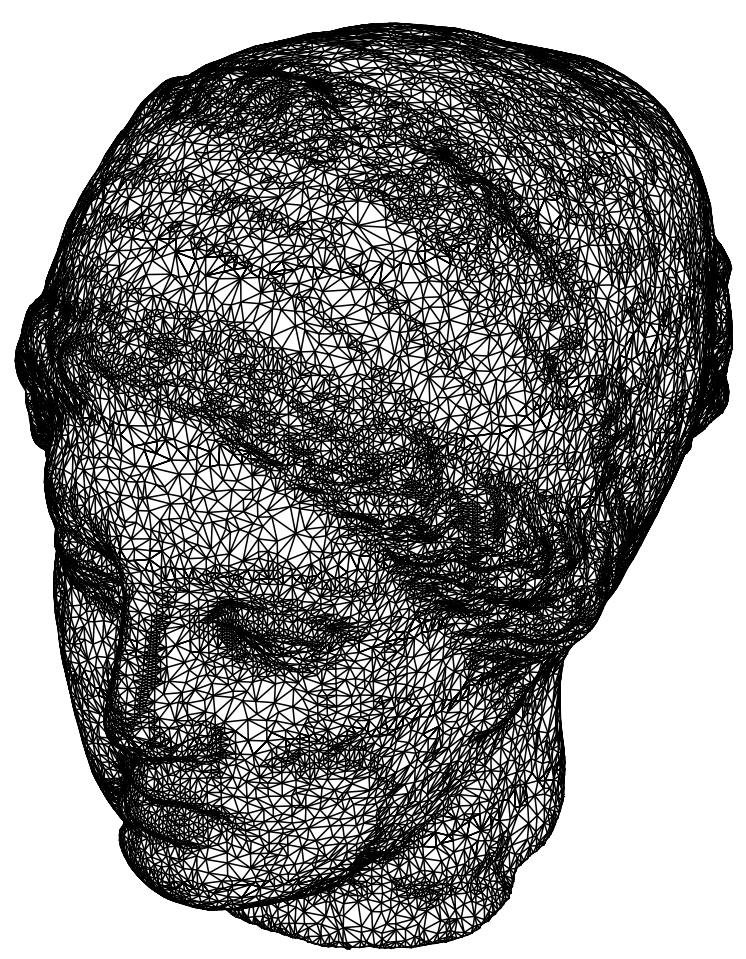

(a)

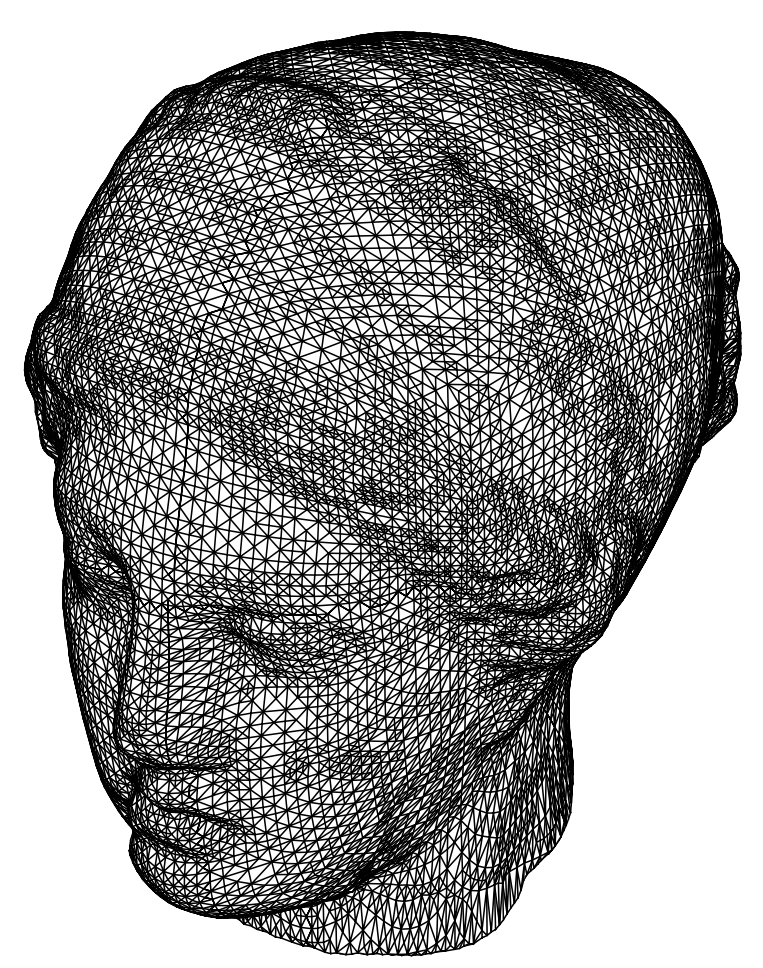

(b)

Fig. 11. (a) Original mesh of Igea obtained from http://www.cyberware.com/ and (b) surface triangulation obtained with the 3-D mesh generator 


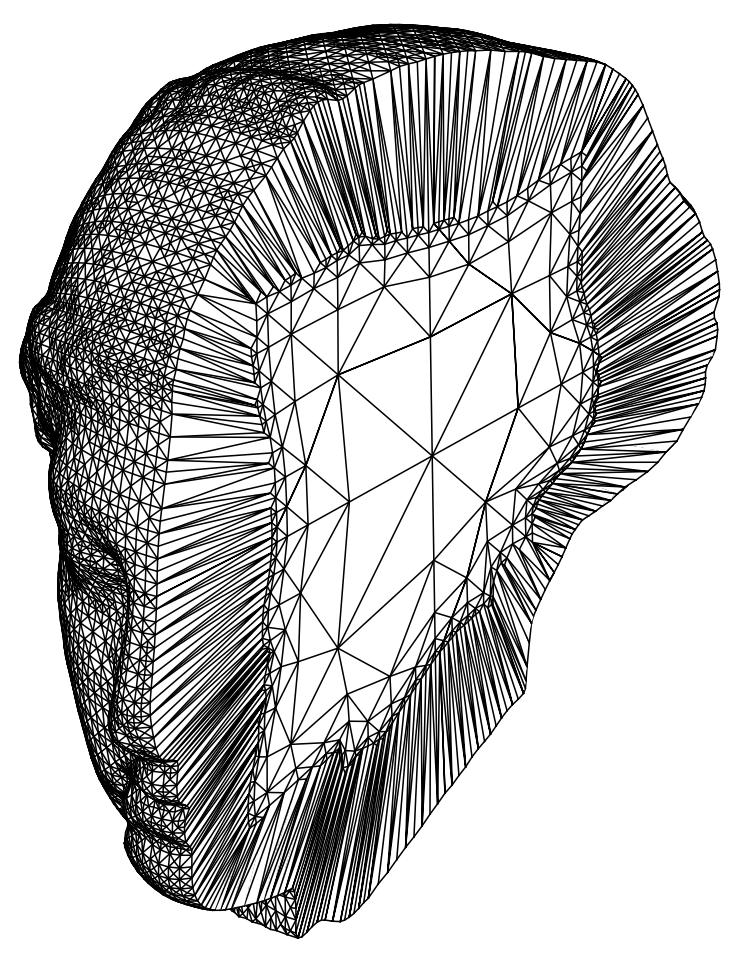

(a)

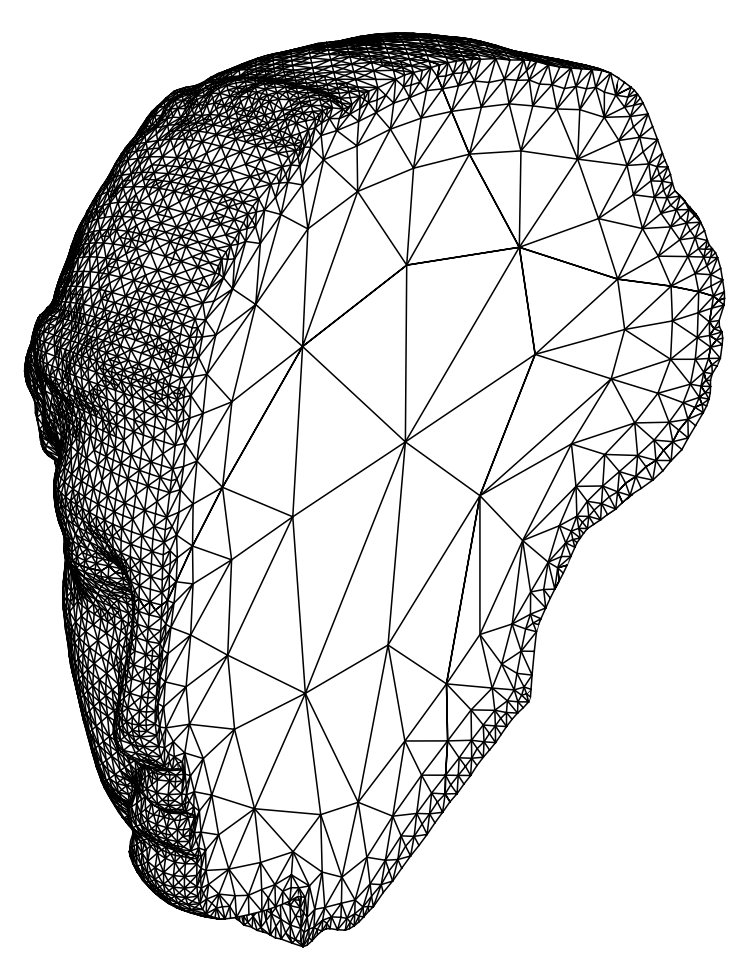

(b)

Fig. 12. Surface triangulation and inner view of the brain of Igea before (a) and after optimization (b) 
fixed, the mesh generation procedure is fully automatic. In future works, we will develop a special CAD package for more general input object. Specifically, object surface patches should be defined by using meccano surfaces as parametric spaces.

The mesh generation technique is based on sub-processes (subdivision, projection, optimization) which are not in themselves new, but the overall integration using a simple shape as starting point is an original contribution of this paper and it has some obvious performance advantages. We have also introduced a generalized derefinement condition for a simple approximation of surfaces. Finally, another interesting property of the new mesh generation strategy is that it automatically fixes the boundary between materials and achieves a good mesh adaption to the geometrical characteristics of the domain.

\section{Acknowledgments}

This work has been supported by the Spanish Government, "Secretaría de Estado de Universidades e Investigación", "Ministerio de Educación y Ciencia", and FEDER, grant contracts: CGL2004-06171-C03, CGL2007-65680-C03 and CGL2008-06003C03. We would also like to thank the authors of ALBERTA [29] for the code availability in internet [30] and for their suggestions.

\section{References}

[1] M. S. Bazaraa, H. D. Sherali, C. M. Shetty, Nonlinear Programing: Theory and Algorithms, John Wiley and Sons Inc., New York, 1993.

[2] G. F. Carey, Computational Grids: Generation, Adaptation and Solution Strategies, Taylor \& Francis, Washington, 1997.

[3] G. F. Carey, A perspective on adaptive modeling and meshing (AM\&M), Comput. Meth. Appl. Mech. Eng. 195 (2006) 214-235.

[4] J. M. Cascón, R. Montenegro, J. M. Escobar, E. Rodríguez, G. Montero, A new meccano technique for adaptive 3-d triangulation, in: Proc. of the 16th International Meshing Roundtable, Springer, Berlin, 2007, pp. 103-120.

[5] J. M. Escobar, R. Montenegro, Several aspects of three-dimensional Delaunay triangulation, Adv. Eng. Soft. 27 (1996) 27-39.

[6] J. M. Escobar, G. Montero, R. Montenegro, E. Rodríguez, An algebraic method for smoothing surface triangulations on a local parametric space, Int. J. Num. Meth. Eng. 66 (2006) 740-760. 
[7] J. M. Escobar, E. Rodríguez, R. Montenegro, G. Montero, J. M. González-Yuste, Simultaneous untangling and smoothing of tetrahedral meshes, Comput. Meth. Appl. Mech. Eng. 192 (2003) 2775-2787.

[8] L. Ferragut, R. Montenegro, A. Plaza, Efficient refinement/derefinement algorithm of nested meshes to solve evolution problems, Comm. Num. Meth. Eng. 10 (1994) $403-$ 412.

[9] L. A. Freitag, P. M. Knupp, Tetrahedral mesh improvement via optimization of the element condition number, Int. J. Num. Meth. Eng. 53 (2002) 1377-1391.

[10] L. A. Freitag, P. Plassmann, Local optimization-based simplicial mesh untangling and improvement, Int. J. Num. Meth. Eng. 49 (2000) 109-125.

[11] P. J. Frey, P. L. George, Mesh Generation, Hermes Science Publishing, Oxford, 2000.

[12] P. L. George, H. Borouchaki, Delaunay Triangulation and Meshing: Application to Finite Elements, Editions Hermes, Paris, 1998.

[13] P. L. George, F. Hecht, E. Saltel, Automatic mesh generation with specified boundary, Comput. Meth. Appl. Mech. Eng. 92 (1991) 269-288.

[14] J. M. González-Yuste, R. Montenegro, J. M. Escobar, G. Montero, E. Rodríguez, Local refinement of 3-d triangulations using object-oriented methods, Adv. Eng. Soft. 35 (2004) 693-702.

[15] P. M. Knupp, Achieving finite element mesh quality via optimization of the jacobian matrix norm and associated quantities. Part II - A frame work for volume mesh optimization and the condition number of the jacobian matrix, Int. J. Num. Meth. Eng. 48 (2000) 1165-1185.

[16] P. M. Knupp, Algebraic mesh quality metrics, SIAM J. Sci. Comput. 23 (2001) 193218.

[17] I. Kossaczky, A recursive approach to local mesh refinement in two and three dimensions, J. Comput. Appl. Math. 55 (1994) 275-288.

[18] R. Löhner, J. D. Baum, Adaptive h-refinement on 3-d unstructured grids for transient problems, Int. J. Num. Meth. Fluids 14 (1992) 1407-1419.

[19] J. Maubach, Local bisection refinement for n-simplicial grids generated by reflection, SIAM J. Sci. Comput. 16 (1995) 210-227.

[20] W. F. Mitchell, A comparison of adaptive refinement techniques for elliptic problems, ACM Trans. Math. Soft. 15 (1989) 326-347.

[21] R. Montenegro, J. M. Cascón, J. M. Escobar, E. Rodríguez, G. Montero, Implementation in alberta of an automatic tetrahedral mesh generator, in: Proc. of the 15th International Meshing Roundtable, Springer, Berlin, 2006, pp. 325-338.

[22] R. Montenegro, J. M. Escobar, G. Montero, E. Rodríguez, Quality improvement of surface triangulations, in: Proc. of the 14th International Meshing Roundtable, Springer, Berlin, 2005, pp. 469-484. 
[23] R. Montenegro, G. Montero, J. M. Escobar, E. Rodríguez, Efficient strategies for adaptive 3-d mesh generation over complex orography, Neural, Parallel \& Scientific Computation 10 (2002) 57-76.

[24] R. Montenegro, G. Montero, J. M. Escobar, E. Rodríguez, J. M. González-Yuste, Tetrahedral mesh generation for environmental problems over complex terrains, in: Lecture Notes in Computer Science, vol. 2329, Springer, Berlin, 2002, pp. 335-344.

[25] G. Montero, R. Montenegro, J. M. Escobar, E. Rodríguez, J. M. González-Yuste, Velocity field modelling for pollutant plume using 3-d adaptive finite element method, in: Lecture Notes in Computer Science, vol. 3037, Springer, Berlin, 2004, pp. 642 645.

[26] G. Montero, E. Rodríguez, R. Montenegro, J. M. Escobar, J. M. González-Yuste, Genetic algorithms for an improved parameter estimation with local refinement of tetrahedral meshes in a wind model, Adv. Eng. Soft. 36 (2005) 3-10.

[27] M. C. Rivara, A grid generator based on 4-triangles conforming. Mesh-refinement algorithms, Int. J. Num. Meth. Eng. 24 (1987) 1343-1354.

[28] M. C. Rivara, C. Levin, A 3-d refinement algorithm suitable for adaptive multigrid techniques, J. Comm. Appl. Numer. Meth. 8 (1992) 281-290.

[29] A. Schmidt, K. G. Siebert, Design of Adaptive Finite Element Software: The Finite Element Toolbox ALBERTA, vol. 42 of Lecture Notes in Computer Science, Springer, Berlin, 2005.

[30] A. Schmidt, K. G. Siebert, D. Köster, O. Kriessl, C. J. Heine, ALBERTA - an adaptive hierarchical finite element toolbox (2007).

URL http: / /www .alberta-fem.de/

[31] J. F. Thompson, B. Soni, N. Weatherill, Handbook of Grid Generation, CRC Press, London, 1999.

[32] C. T. Traxler, An algorithm for adaptive mesh refinement in $\mathrm{n}$ dimensions, Computing 59 (1997) 115-137. 